\title{
ANÁLISE SENSORIAL DESCRITIVA QUANTITATIVA DE GELEIA DE GABIROBA (Camponesia cambessedeana)
}

Quantitative Descriptive Sensory Analysis of Gabiroba Jelly (Camponesia cambessedeana)

Análisis Sensorial Descriptivo Cuantitativo dela Jalea de Gabiroba (Camponesia cambessedeana)

\section{Cecília Marques Tenório Pereira ${ }^{* 1}$, Diana Lopes da Silva², Gabriela Fonseca Leal ${ }^{2}$, Glêndara Aparecida de Souza Martins ${ }^{2}$, Caroline Roberta Freitas Pires ${ }^{1}$}

${ }^{1}$ Laboratório de Tecnologia de Alimentos, Curso de Nutrição, Universidade Federal do Tocantins, Palmas, Brasil.

${ }^{2}$ Laboratório de Cinética e Modelagem de Processos, Curso de Engenharia de Alimentos, Universidade Federal do Tocantins, Palmas, Brasil.

*Correspondência: Laboratório de Tecnologia de Alimentos, Universidade Federal do Tocantins, Av. NS 15, 109 Norte, Palmas, Tocantins, Brasil.CEP:77.010-090.e-mail cecilia.tenorio@mail.uft.edu.br

\section{RESUMO}

As frutas e vegetais são fontes de vitaminas antioxidantes ( $\mathrm{C}$ e E), compostos fenólicos e carotenos, sendo a gabiroba (Camponesia cambessedeana) uma delas, podendo ser consumida na forma in natura, como polpa de fruta, e na forma processada como sorvetes, doces e geleias. O objetivo desse trabalho foi realizar o perfil sensorial da geleia de gabiroba. Foram desenvolvidas 11 formulações, avaliadas quanto aos atributos: aparência, sabor, textura, impressão global e intenção de compra, e a partir da análise desses resultados, utilizou-se a melhor formulação para realizar a Análise Descritiva Quantitativa. A formulação 8, com razão polpa/açúcar 40/60, sem adição de ácido cítrico e pectina, apresentou valor significativamente maior que as demais amostras em relação a todos os atributos pelos testes sensoriais afetivos. A equipe sensorial definiu os termos descritivos e os materiais de referências para geleia de gabiroba. A aplicação da Análise Descritiva Quantitativa na avaliação sensorial de geleia de gabiroba mostrou que as amostras apresentam cor amarelo-amarronzada, brilho característico, corpo, aroma doce, sabor amargo e sabor de gabiroba. Os tributos, sabor adstringente, aroma de gabiroba e aroma ácido, apresentaram baixa intensidade.

Palavras-chave: Camponesia cambessedeana. Análise Descritiva Quantitativa. Análise Sensorial

\section{ABSTRACT}

Fruits and vegetables are sources of antioxidant vitamins ( $C$ and E), phenolic compounds and carotenes, being gabiroba (Camponesia cambessedeana) one of them, which can be consumed in its natural form as fruit pulp and processed as ice cream, Sweets and jellies. The objective of this work was to perform the sensory profile of gabiroba jelly. Eleven formulations were developed, evaluated for the attributes: appearance, taste, texture, overall impression and purchase intention, and from the analysis of these results, the best formulation was used to perform the Quantitative Descriptive Analysis. Formulation 8, with a 40/60 pulp/sugar ratio, without the addition of citric acid and pectin, presented a significantly higher value than the other samples in relation to all attributes by affective sensory tests. The sensory team defined the descriptive terms and reference materials for gabiroba jelly. The application of the Quantitative Descriptive Analysis to the sensory evaluation of gabiroba jelly showed that the samples have a brownish-yellow color, characteristic brightness, body, sweet aroma, bitter taste and gabiroba flavor. The tributes, astringent flavor, gabiroba aroma and acid aroma presented low intensity.

Keywords: Camponesia cambessedeana. Quantitative Descriptive Analysis. Sensory analysis 


\section{RESUMEN}

Las frutas y verduras son fuentes de vitaminas antioxidantes (C y E), compuestos fenólicos y carotenos, gabiroba (Camponesia cambessedeana) es una de ellas, puede consumirse em forma fresca, como pulpa de fruta y processarce como helado, mermelados y jaleas. El objetivo de este trabajo fue realizar el perfil sensorial de la jalea de gabiroba. Se desarrollaron once formulaciones, evaluadas para los atributos: apariencia, sabor, textura, impresión general e intención de compra, y del análisis de estos resultados, se utilizó la mejor formulación para realizar el Análisis Descriptivo Cuantitativo. La formulación 8, con una relación pulpa/azúcar 40/60, sin la adición de ácido cítrico y pectina, presentó un valor significativamente más alto que las otras muestras en relación con todos los atributos mediante pruebas sensoriales afectivas. El equipo sensorial definió los términos descriptivos y los materiales de referencia para la jalea de gabiroba. La aplicación del Análisis Descriptivo Cuantitativo en la evaluación sensorial de la jalea de gabiroba mostró que las muestras tienen un color amarillo parduzco, brillo característico, cuerpo, aroma dulce, sabor amargo y sabor a gabiroba. Los homenajes, el sabor astringente, el aroma de gabiroba y el aroma ácido presentaron baja intensidad.

Descriptores: Camponesia cambessedeana. Análisis Descriptivo Cuantitativo. Análisis sensorial

\section{INTRODUÇÃO}

O bioma do Cerrado tem uma área de 2,0 milhões de $\mathrm{km}^{2}$, aproximadamente $24 \%$ do território nacional, abrangendo os estados Mato Grosso, Piauí, Bahia, Maranhão, Goiás, Tocantins, Mato Grosso do Sul, Minas Gerais e Distrito Federal (SOARES et. al., 2017) com ênfase para as espécies frutíferas exóticas detentoras de características sensoriais peculiares.

Essas frutas são constituídas por uma fonte de compostos com propriedades benéficas à saúde, podendo estimular seu pela indústria farmacêutica e de alimentos para o desenvolvimento de novos produtos (SIQUEIRA et. al., 2013), a gabiroba (Camponesia cambessedeana) uma delas. A coloração do fruto é amarelada ou alaranjada, no estádio maduro, com aroma cítrico agradável, polpa suculenta e possui formato globoso, apresentando diâmetros entre 15 e 20 mm (ALBUQUERQUE, 2016).

A polpa da gabiroba pode ser utilizada na obtenção de geleias, doces, sucos e sorvetes (GUIMARÃES, 2016). As geleias são obtidas através da mistura de seus ingredientes em proporções devidas, preparadas a partir da ebulição de polpa de fruta juntamente com açúcar, ácido e agente geleificante (BASU; SHIVHARE, 2010).
A geleia pode ser comum, quando preparadas numa proporção de 40 partes de frutas para 60 partes de açúcar, ou extra, quando preparadas numa proporção de 50 partes de frutas para 50 partes de açúcar (BRASIL, 2005), classificadas em simples (única espécie de fruta) e mistas, (mais de uma espécie de fruta). A calda deve ser concentrada até o ${ }^{\circ}$ Brix suficiente para que ocorra geleificação durante o resfriamento (Instituto Adolfo Lutz, 2005).

No desenvolvimento de novos produtos, é indispensável que alguns parâmetros como a cor, forma, sabor, odor, textura, consistência e a interação entre os diferentes componentes sejam avaliados e aprimorados, a fim de se obter um produto de qualidade e com boa aceitação (ARAUJO et. al., 2012).

A Análise Descritiva Quantitativa (ADQ) é um dos métodos mais utilizados e permite a descrição detalhada do produto em relação a atributos sensoriais, ou intenção de comparar vários produtos em relação às suas diferenças sensoriais (LAWLESS; HEYMANN, 2010). Segundo Meilgaard et al. (2006), é comum utilizar a ADQ para melhorar e desenvolver novos produtos, assim como para o controle de qualidade, além de vida de prateleira. Os testes sensoriais de 
aceitação têm por objetivo avaliar se os consumidores gostam ou desgostam da amostra avaliada.

Este trabalho tem como objetivo traçar o perfil sensorial da geleia de gabiroba.

\section{MATERIAIS E MÉTODO}

Foram desenvolvidas 11 formulações de geléia de gabiroba de acordo com as concentrações de ácido cítrico, razão polpa/açúcar e albedo de maracujá apresentados na tabela 1. Para a produção da geleia utilizou-se a polpa de gabiroba congelada, comprada em uma indústria no Estado de Goiás. Utilizou-se o albedo de maracujá como fonte de pectina, conforme descrito por Silva et al. (2012).

Tabela 1 - Concentrações para as formulações da geleia de gabiroba.

\begin{tabular}{cccc}
\hline Amostra & \multicolumn{3}{c}{ Concentrações } \\
\hline & $\begin{array}{c}\text { Ác. } \\
\text { Cítrico }\end{array}$ & $\begin{array}{c}\text { Razão } \\
\text { polpa/açúcar }\end{array}$ & Albedo \\
$\mathbf{1}$ & 1 & $60 / 40$ & 3 \\
$\mathbf{2}$ & 0 & $40 / 60$ & 3 \\
$\mathbf{3}$ & 1 & $40 / 60$ & 3 \\
$\mathbf{4}$ & 0 & $60 / 40$ & 3 \\
$\mathbf{5}$ & 1 & $60 / 40$ & 0 \\
$\mathbf{6}$ & 0 & $60 / 40$ & 0 \\
$\mathbf{7}$ & 1 & $40 / 60$ & 0 \\
$\mathbf{8}$ & 0 & $40 / 60$ & 0 \\
$\mathbf{9}$ & 0,5 & $50 / 50$ & 1,5 \\
$\mathbf{1 0}$ & 0,5 & $50 / 50$ & 1,5 \\
$\mathbf{1 1}$ & 0,5 & $50 / 50$ & 1,5 \\
\hline
\end{tabular}

A pesquisa foi submetida previamente ao Comitê de Ética em Pesquisa da Universidade Federal do Tocantins (UFT), o qual foi aprovado com o Certificado de Apresentação para a Apreciação Ética número 93357718.3.0000.5519 e parecer número 3.096.189.

Análises microbiológicas (bolores, leveduras, estafilococos, coliformes totais e salmonela) da geleia de gabiroba foram realizadas em estudo complementar, para verificar se houve crescimento microbiológico, como não houve, deu-se o prosseguimento com as análises sensoriais.

\section{Análise Sensorial Afetiva}

As 11 formulações foram avaliadas quanto a aceitação para os atributos aroma, sabor, textura, impressão global (STONE; SIDEL, 2004) e intenção de compra (SCHUTZ, 1965) para a geleia de gabiroba.

As amostras de geleia foram servidas conforme metodologia de Acosta et. al. (2008). Os testes de aceitação e intenção de compra foram realizados no Complexo Laboratorial, do curso de Nutrição, com 36 provadores não treinados, acadêmicos dos cursos da UFT, de ambos os sexos e idades entre 18 e 40 anos.

A partir da análise desses resultados, a amostra que apresentar a melhor avaliação quanto a aceitação e intenção de compra será avaliada quanto a Análise Sensorial Descritiva.

\section{Análise Sensorial Descritiva}

Para seleção da equipe, foram convidados acadêmicos dos cursos da UFT, de ambos os sexos e idades entre 18 e 40 anos, que preencheram uma ficha de cadastro.

Pré-selecionou-se julgadores pré-avaliados pelo teste de reconhecimento de aromas, no qual os provadores deveriam acertar $70 \%$ dos aromas com grau de dificuldade normal. O teste de gosto básico, foi representado por 6 séries de três amostras, utilizando soluções aquosas de $\mathrm{NaCl}$, sacarose e ácido cítrico, aprovando os provadores que obtiveram $100 \%$ das respostas. No teste triangular de aroma e gosto, representado por 6 séries de três amostras, com formulações diferentes, sendo duas amostras iguais e uma diferente, foram aprovados os provadores que obtiveram $60 \%$ de acertos. 
Após a seleção dos julgadores, estes foram treinados para conseguir traçar o perfil sensorial da geleia com maior aceitação. As geleias foram colocadas em temperatura ambiente, na posição central da mesa, permitindo a interação do grupo. A sessão foi conduzida de forma a obter o máximo de descritores que pudessem traçar o perfil sensorial.

\section{Treinamento da equipe}

Os termos que expressaram o mesmo significado foram agrupados em um só atributo, os termos poucos utilizados foram eliminados. No final das sessões, foi gerada uma lista de termos descritivos de Cor; Brilho; Aroma; Sabor e Textura característica, com os respectivos extremos da escala utilizada.

Os provadores avaliaram a intensidade de cada atributo identificado, na descrição da terminologia das amostras utilizando uma escala hedônica não estruturada de $9 \mathrm{~cm}$, ancorada nos extremos com termos definidos pela equipe. As amostras da geleia de gabiroba foram avaliadas em oito repetições pelos provadores selecionados, de forma individual, utilizando água mineral para lavar o palato entre uma amostra e outra.

\section{Análise estatística}

Os dados obtidos na análise sensorial foram analisados através da análise de variância (ANOVA) utilizando o programa estatístico SISVAR (FERREIRA, 2000) e, identificando diferenças significativas entre as médias $(\mathrm{p} \leq 0,05)$, as mesmas foram submetidas ao teste de Tukey a um nível de significância de 5\%.

\section{RESULTADOS E DISCUSSÃO}

\section{Análise Sensorial Afetiva}

A tabela 02 apresenta os valores médios obtidos da avaliação sensorial das onze formulações da geleia de gabiroba. Pode-se observar que a amostra 8 apresentou valor significativamente maior que as demais amostras em relação a todos os atributos.

As amostras 3, 6 e 9 não se diferiram entre si estatisticamente em relação ao atributo aroma. Já em relação ao atributo sabor, a amostra 6 foi a que obteve o segundo melhor valor. Para o atributo textura, as amostras 4 e 5 não se diferiram da 8. Com relação ao atributo impressão global, a amostra 8 também apresentou valor maior, seguido pela amostra 6, o menor valor foi apresentado pela amostra 2.

Freitas et al., (2008) relataram que a baixa aceitação da geleia de gabiroba, pode ser atribuída ao seu sabor amargo e o desconhecimento dos provadores

Tabela 2 - Valores médios dos atributos sensoriais

\begin{tabular}{cccccc}
\hline Amostra & Aroma & Sabor & Textura & Impressão Global & Intenção Compra \\
\hline $\mathbf{1}$ & $5,75^{\mathrm{c}}$ & $4,50^{\mathrm{f}}$ & $3,50^{\mathrm{d}}$ & $4,00^{\mathrm{e}}$ & $300^{\mathrm{c}}$ \\
$\mathbf{2}$ & $5,50^{\mathrm{d}}$ & $3,00^{\mathrm{h}}$ & $2,50^{\mathrm{f}}$ & $3,00^{\mathrm{g}}$ & $2,00^{\mathrm{e}}$ \\
$\mathbf{3}$ & $6,00^{\mathrm{b}}$ & $3,50^{\mathrm{g}}$ & $2,00^{\mathrm{g}}$ & $3,50^{\mathrm{f}}$ & $3,00^{\mathrm{c}}$ \\
$\mathbf{4}$ & $5,00^{\mathrm{e}}$ & $4,50^{\mathrm{f}}$ & $7,00^{\mathrm{a}}$ & $3,50^{\mathrm{f}}$ & $2,00^{\mathrm{e}}$ \\
$\mathbf{5}$ & $5,00^{\mathrm{e}}$ & $5,50^{\mathrm{d}}$ & $7,00^{\mathrm{a}}$ & $5,00^{\mathrm{c}}$ & $3,00^{\mathrm{c}}$ \\
$\mathbf{6}$ & $6,00^{\mathrm{b}}$ & $6,50^{\mathrm{b}}$ & $4,50^{\mathrm{b}}$ & $5,50^{\mathrm{b}}$ & $3,50^{\mathrm{b}}$ \\
$\mathbf{7}$ & $5,50^{\mathrm{d}}$ & $5,00^{\mathrm{e}}$ & $2,50^{\mathrm{f}}$ & $4,00^{\mathrm{e}}$ & $3,00^{\mathrm{c}}$ \\
$\mathbf{8}$ & $6,50^{\mathrm{a}}$ & $7,00^{\mathrm{a}}$ & $7,00^{\mathrm{a}}$ & $6,50^{\mathrm{a}}$ & $4,00^{\mathrm{a}}$ \\
$\mathbf{9}$ & $6,00^{\mathrm{b}}$ & $5,50^{\mathrm{d}}$ & $4,50^{\mathrm{b}}$ & $5,00^{\mathrm{c}}$ & $4,00^{\mathrm{a}}$ \\
\hline
\end{tabular}




\begin{tabular}{llllll}
\hline $\mathbf{1 0}$ & $4,50^{\mathrm{f}}$ & $6,00^{\mathrm{c}}$ & $3,00^{\mathrm{e}}$ & $4,50^{\mathrm{d}}$ & $2,50^{\mathrm{d}}$ \\
$\mathbf{1 1}$ & $4,50^{\mathrm{f}}$ & $6,00^{\mathrm{c}}$ & $4,00^{\mathrm{c}}$ & $4,00^{\mathrm{e}}$ & $2,00^{\mathrm{e}}$ \\
\hline
\end{tabular}

As médias das amostras acompanhadas pela mesma letra, na mesma linha, não diferem entre si (\%5 de significância) pelo teste de Tukey. Fonte: dados da pesquisa (2019).

dos sabores característicos de frutos do cerrado.

triangulares de gostos e aromas, dos 19 provadores que

De acordo com os resultados, a amostra 8 obteve as maiores médias para os atributos impressão global e intenção de compra. participaram apenas 13 permaneceram na equipe sensorial da ADQ.

Foram realizados encontros, reunindo todos os

\section{Análise Descritiva Quantitativa}

No teste de aromas, dos 24 participantes, 19 alcançaram o mínimo de acertos. Para os testes de gostos básicos, participaram 19 provadores, e todos obtiveram o critério de aprovação. Nos testes participantes aprovados nos testes prévios para a condução da análise sensorial. Os materiais de referência e a definição de cada termo descritivo foram colocados à disposição dos provadores. A definição de cada termo descritivo foi desenvolvida pela equipe sensorial, representada na Tabela 3.

Tabela 3 - Definição dos termos descritivos e materiais de referências para geleia de gabiroba

\begin{tabular}{cccc}
\hline & Descritores & Definição & Referência \\
\hline \multirow{2}{*}{ Aparência } & $\begin{array}{c}\text { Cor Amarelo- } \\
\text { Amarronzada }\end{array}$ & $\begin{array}{c}\text { Tonalidade da cor amarela variando de } \\
\text { amarelo claro ao amarelo escuro. }\end{array}$ & Fraca: marmelada / Forte: calda de caramelo \\
Aroma & Gabiroba & Intensidade aroma de gabiroba & Fraca: rapadura / Forte: polpa gabiroba \\
& Doce & Intensidade aroma doce & Fraca: rapadura / Forte: polpa gabiroba \\
Sabor & Gabiroba & Intensidade sabor de gabiroba & Fraca: rapadura / Forte: polpa gabiroba \\
Textura & Corpo & Intensidade sabor amargo & Fraca: rapadura / Forte: polpa gabiroba \\
\hline
\end{tabular}

Cada provador recebeu oito amostras de geleia, juntamente com as fichas do teste de escala hedônica não estruturada para o julgamento dos sensoriais, apenas 8 deles foram selecionados pela sua capacidade de repetibilidade, reprodutibilidade e poder discriminativo.

atributos. Dos 13 participantes que fizeram os testes

Tabela 4 - Valores médios das notas atribuídas por cada provador para cada atributo sensorial avaliado

\begin{tabular}{ccccccccccc}
\hline & \multicolumn{2}{c}{ Aparência } & \multicolumn{3}{c}{ Aroma } & & \multicolumn{2}{c}{ Sabor } & \multicolumn{2}{c}{ Textura } \\
\hline Provad. & Cor & Brilho & Gabiroba & Ácido & Doce & Gabiroba & Amargo & Adstring & Corpo \\
P1 & $6,72 \pm 0,23$ & $7,8 \pm 0,21$ & $4,3 \pm 0,16$ & $2,1 \pm 0,08$ & $6,6 \pm 0,12$ & $5,6 \pm 0,31$ & $5,22 \pm 0,3$ & $1,97 \pm 0,32$ & $6,55 \pm 0,32$ \\
& & & & & & & & & & \\
P2 & $6,65 \pm 0,19$ & $7,47 \pm 0,23$ & $4,02 \pm 0,12$ & $2,5 \pm 0,34$ & $6,57 \pm 0,16$ & $5,77 \pm 0,32$ & $6,05 \pm 0,45$ & $2,87 \pm 0,26$ & $6,15 \pm 0,13$ \\
P3 & $6,45 \pm 0,22$ & $7,57 \pm 0,14$ & $3,32 \pm 0,09$ & $2,57 \pm 0,19$ & $6,6 \pm 0,15$ & $5,47 \pm 0,27$ & $6,27 \pm 0,23$ & $2,27 \pm 0,19$ & $6,32 \pm 0,26$ \\
P4 & $6,4 \pm 0,13$ & $7,35 \pm 0,08$ & $4,52 \pm 0,07$ & $2,02 \pm 0,15$ & $6,75 \pm 0,21$ & $5,2 \pm 0,13$ & $5,52 \pm 0,31$ & $2,25 \pm 0,24$ & $6,4 \pm 0,32$ \\
P5 & $6,85 \pm 0,08$ & $7,47 \pm 0,14$ & $4,62 \pm 0,31$ & $2,4 \pm 0,09$ & $6,72 \pm 0,17$ & $5,15 \pm 0,14$ & $5,12 \pm 0,21$ & $2,22 \pm 0,43$ & $6,55 \pm 0,41$ \\
& & & & & & & & & &
\end{tabular}




\begin{tabular}{cccccccccc}
\hline P6 & $6,4 \pm 0,13$ & $7,52 \pm 0,13$ & $4,65 \pm 0,14$ & $2,65 \pm 0,21$ & $7,1 \pm 0,16$ & $6,02 \pm 0,19$ & $5,07 \pm 0,13$ & $2,42 \pm 0,32$ & $6,22 \pm 0,16$ \\
P7 & $6,32 \pm 0,17$ & $7,3 \pm 0,15$ & $4,75 \pm 0,16$ & $2,52 \pm 0,16$ & $6,4 \pm 0,16$ & $5,95 \pm 0,21$ & $5,8 \pm 0,24$ & $1,85 \pm 0,21$ & $6,85 \pm 0,49$ \\
P8 & $6,67 \pm 0,21$ & $7,45 \pm 0,31$ & $3,85 \pm 0,11$ & $2,65 \pm 0,08$ & $6,75 \pm 0,21$ & $5,52 \pm 0,11$ & $5,1 \pm 0,19$ & $1,7 \pm 0,54$ & $6,47 \pm 0,25$ \\
Média \pm SD & $6,55 \pm 0,19$ & $7,49 \pm 0,15$ & $4,25 \pm 0,49$ & $2,42 \pm 0,24$ & $6,68 \pm 0,21$ & $5,58 \pm 0,32$ & $5,52 \pm 0,47$ & $2,19 \pm 0,37$ & $6,44 \pm 0,22$ \\
\hline
\end{tabular}

A tabela 4 apresenta os valores médios das notas atribuídas por cada provador para cada atributo sensorial avaliado. De acordo com a tabela no atributo cor os valores variaram entre 6,32 a 6,85 , com média de 6,55, indicando uma coloração mais amarronzada. De acordo com Guizilini (2010) com o aquecimento da polpa, há possibilidade de modificação da coloração da geleia, em decorrência da degradação dos carotenóides ou da caramelização dos açúcares.

Quanto ao brilho, observou-se que os valores médios variaram de 7,3 a 7,8. A média da equipe sensorial foi de 7,49, indicando que a geleia apresenta brilho característico, visto que as médias foram elevadas. Dentre os principais atributos de qualidade de uma geleia temos: consistência que, quando extraída do recipiente, é capaz de se manter em estado semissólido adequado e cor brilhante (RABABAH et al., 2012).

Para o aroma de gabiroba as médias variaram entre 3,32 a 4,75, com média da equipe sensorial de 4,25. Estes resultados mostram que os valores médiossão baixos, indicando uma leve sensação do aroma de gabiroba. Quanto ao aroma ácido as médias variaram entre 2,02 a 2,65. As médias para o aroma doce se sobressaíram em relação aos demais aromas, variando entre 6,4 a 7,1 . Valores elevados para o aroma doce pode ser justificado pela presença de açúcar nas formulações, que diante da concentração de $67,5^{\circ}$ Brix imprimem o aroma adocicado.

Os alimentos consumidos contêm substâncias aromáticas no seu estado in natura ou formadas através da cocção do alimento. O cheiro dos alimentos pode ser o principal argumento para comê-los e suas características dependem mais do aroma do que do gosto, ambos constituem o sabor (AROMAS, 2015).

Para o sabor de gabiroba, a média entre os provadores variaram entre 5,15 a 6,02 com uma média da equipe sensorial de 5,58. De acordo com Kinupp (2007) a gabiroba apresenta um sabor doce e/ou acidulado que são próprias para a produção de polpa concentrada e congelada. Para o sabor amargo o valor médio da equipe sensorial foi de 5,52. Este valor encontra-se elevado, indicando a presença de um amargor no produto final, corroborando com os resultados encontrados por Freitas et al., (2008) que atribuiu a baixa aceitação do produto ao amargor da geleia. Já para o sabor adstringente, observou-se que os valores médios variaram de 1,7 a 2,87 com uma média da equipe sensorial de 2,19. Guizilini (2010) citou que a polpa retirada do fruto sem semente e casca é menos adstringente em comparação a polpa com o fruto integral.

Quanto ao corpo da geleia, associada à textura da mesma, observou-se que a média variou entre 6,15 a 6,85, com valores médios da equipe sensorial de 6,44, sendo considerada pelos provadores como muito encorpada. Este fato pode ser atribuído às características de acidez do fruto que na presença de açúcar favorece a formação de um gel rígido.

Para melhor visualização foi construído o gráfico de aranha que apresenta os valores médios para os atributos sensoriais. De acordo com a figura 4 a amostra apresenta uma cor amarelo-amarronzada mais acentuada, além do brilho, corpo, aroma doce, sabor amargo e sabor de gabiroba mais intenso. 
Figura 4 - Gráfico de aranha com os valores médios para cada atributo

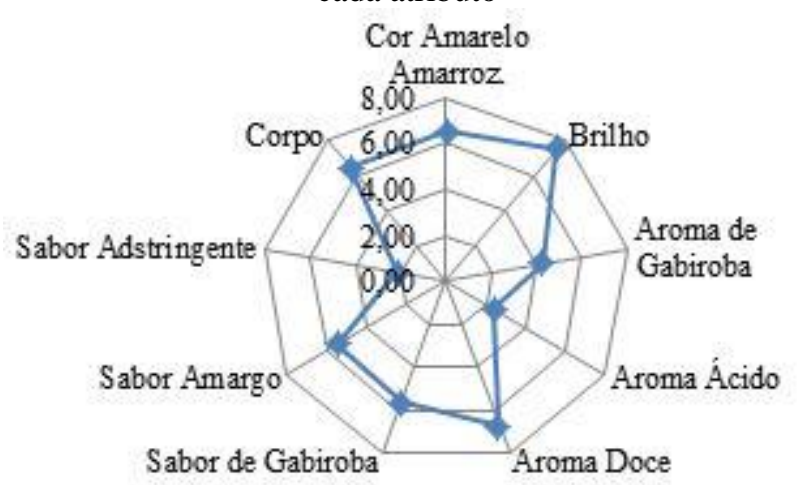

Fonte: dados da pesquisa (2019).

\section{CONCLUSÃO}

A amostra 8, com razão polpa/açúcar 40/60, sem adição de ácido cítrico e albedo, apresentou melhor avaliação pelos testes sensoriais afetivos.

A aplicação da Análise Descritiva Quantitativa na avaliação sensorial da geleia de gabiroba foi descrita por nove atributos sensoriais, sendo os mais intensos: cor amarelo-amarronzado, brilho, aroma doce, sabor de gabiroba, sabor amargo, e corpo.

\section{AGRADECIMENTO}

A toda equipe do Laboratório de Cinética e Modelagem de Processos - LaCiMP e do Laboratório de Tecnologia de Alimentos/Nutrição.

Todos os autores declararam não haver qualquer potencial conflito de interesses referente a este artigo.

\section{REFERÊNCIAS}

ACOSTA, O.; VÍQUEZ, F.; CUBERO, E. Optimization of low calorie mixed fruit jelly by response surface methodology. Food Quality and Preference, Barking, v. 19, n. 1, p. 79-85, 2008.

ALBUQUERQUE, J. S. Propagação vegetativa de guabiroba (Campomanesia xanthocarpa Berg.) pelo método de enxertia. 2016. (Trabalho de conclusão de curso). Curso de Engenharia Florestal, Universidade Federal do Paraná, Curitiba, 2016.
ARAUJO, E. R.; RÊGO, E. R. do; SAPUCAY, M. J. L. C.; RÊGO, M. M. do; SANTOS, R. M. C. dos. Elaboração e análise sensorial de geleia de pimenta com abacaxi. Revista Brasileira de Produtos Agroindustriais, Campina Grande, v. 14, n. 3, p. 233 238, 2012.

AROMAS. Food Ingredients Brazil, n. 33, p. 30-53, 2015.

BASU, S.; SHIVHARE, U. S. Rheological, textural, microstructural and sensory properties of mango jam. Journal of Food Engineering, v.100, p.357-365, 2010.

BRASIL, EMBRAPA. Preparo artesanal de geleias e geleiadas. Pelotas, RS. Junho. 2005.

FERREIRA, D.F. Análises estatísticas por meio do SISVAR para Windows versão 4.0. In... REUNIÃO ANUAL DA REGIÃO BRASILEIRA DA SOCIEDADE INTERNACIONAL DE BIOMETRIA, 45, 2000. Anais... São Carlos, SP: SIB, p. 255-258, 2000 .

FERREIRA, V.L.P.; ALMEIDA, T.C.A.; PETTINELLI, M.L.C.V.; SILVA, M.A.A.P.; CHAVES, J.B.P.; BARBOSA, E.M.M. Análise sensorial: testes discriminativos e afetivos. manual: série qualidade. Campinas, SBCTA, 2000. 127p.

FREITAS, J.B.; CANDIDO, T.L.N; SILVA, M.R. Geleia de gabiroba: avaliação da aceitabilidade e características físicas e químicas, Goiânia, Pesquisa Agropecuária Tropical. v. 38, n. 2, p. 87-94, 2008.

Guimarães, A. C. G. Potencial antioxidante de treze frutos de espécies de ocorrência no cerrado por diferentes metodologias (Tese de doutorado). Universidade Federal de Lavras, Lavras, 2016.

GUIZILINI, L.A. Atividade antioxidante de gabiroba e aplicação da polpa como ingrediente em sorvete. 2010. 93f. Dissertação (Mestrado em Ciência de Alimentos) - Programa de Mestrado e Doutorado em Ciência De Alimentos, Universidade Estadual de Londrina (UEL), Londrina, 2010.

INSTITUTO ADOLFO LUTZ. Normas analíticas do Instituto Adolfo Lutz: Métodos químicos e físicos para análise de alimentos. 4.ed. Instituto Adolfo Lutz, São Paulo, Brasil, 2005. 1018p. 
KINUPP, V. F. Plantas alimentícias não convencionais da região metropolitana de Porto Alegre, RS. 2007. 500f. Tese (Doutorado em Fitotecnia) - Universidade Federal do Rio Grande do Sul, Porto Alegre.

LAWLESS, H.T.; HEYMANN, H. Sensory Evaluation of Food. Principles and Practices. (2sd ed). New York: Springer. 2010.

LISBÔA, G. N.; KINUPP, V. F.; BARROS, I. B. I. Campomanesia xanthocarpa - Gabiroba. In: CORADIN, L.; SIMINSKI, A.; REIS, A. (Ed.). Espécies nativas da flora brasileira de valor econômico atual ou potencial: plantas para o futuro: Região Sul. Brasília, DF: Ministério do Meio Ambiente, 2011. p. 159-162, 226-242.

MEILGAARD, M.; CIVILlE, G. V.; CARR, B. T.; Sensory Evaluation Techniques. 4.ed. Boca Raton: CRC Press, 2006.

RABABAH, T. M., AL-U'DATT, M., ALMAHASNEH, M., YANG, W.; FENG, H., EREIFEJ, K., KILANI, I., ISHMAIS, M. A. Effect of jam processing and storage on phytochemicals and physiochemical properties of cherry at different temperatures. Journal of Food Processing and Preservation, v. 36, p. 1-8, 2012.

SCHUTZ, H.G. A food action rating scale for measuring food acceptance. Journal of Food Science, v.30, n.2, p. 365-374, 1965.

SILVA, D. B. da; SILVA, D. B.; JUNQUEIRA, N. T. V.; ANDRADE, L. R. M. de. Frutas do Cerrado. Brasília, DF: Embrapa Informação Tecnológica, 2001.

SILVA, R.C.S.N.; MINIM, V.P.R.; SIMIQUELI, A.A.; MORAES, L.E.S.; GOMIDE, A.I.; MINIM, L.A. Optimized Descriptive Profile: A rapid methodology for sensory description. Food Quality and Preference, v. 24, n 1, p. 190-200, 2012.
SIQUEIRA, E.M. de A.; ROSA, F.R.; FUSTINONI, A.M.; SANT'ANA, L.P.; ARRUDA, S.F. Brazilian savanna fruits contain higher bioactive compounds content and higher antioxidant activity relative to the conventional red delicious apple. Plos One, Cambridge, v.8, n.8, p.1-7

SOARES, L. V., MELO, R., OLIVEIRA, W. S., SOUZA, P. M., SCHMIELE, M. Brazilian Cerrado fruits and their potential use in bakery products. In $\mathrm{H}$. Lewis (Ed.), Bread: Consumption, cultural significance and health effects (Chap. 5, pp. 125160). New York: Nova Publisher, 2017.

STONE, H.; SIDEL, J. L. Sensory Evaluation Practices. 3a ed. San Diego: Academic Press, 2004. 